\title{
Asymptotic Expansions for Oscillatory Integrals Using Inverse Functions
}

\author{
James N. Lyness and James W. Lottes* \\ Mathematics and Computer Science Division \\ Argonne National Laboratory \\ 9700 S. Cass Ave., Argonne, IL 60439 USA \\ and \\ School of Mathematics \\ University of New South Wales \\ Sydney NSW 2052, Australia.
}

\begin{abstract}
We treat finite oscillatory integrals of the form $\int_{a}^{b} F(x) \exp [i k G(x)] d x$ in which both $F$ and $G$ are real on the real line, are analytic over the open integration interval, and may have algebraic singularities at either or both interval end points. For many of these, we establish asymptotic expansions in inverse powers of $k$. No appeal to the theories of stationary phase or steepest descent is involved. We simply apply theory involving inverse functions and expansions for a Fourier coefficient $\int_{a}^{b} \phi(t) \exp [i k t] d t$. To this end, we have assembled several results involving inverse functions. Moreover, we have derived a new asymptotic expansion for this integral, valid when $\phi(t)=\sum a_{j} t^{\sigma_{j}}$.
\end{abstract}

\section{Introduction}

The purpose of this paper is to present a theory with which one may construct an asymptotic expansion in inverse powers of $k$ for integrals of the form

$$
I_{0}=\int_{a}^{b} F(x) \exp [i k G(x)] d x .
$$

This theory can be applied to functions of the form

$$
F(x)=(x-a)^{\alpha}(b-x)^{\beta} \tilde{F}(x) ; \quad G(x)=(x-a)^{\alpha^{\prime}}(b-x)^{\beta^{\prime}} \tilde{G}(x) ; \quad \alpha, \beta, \alpha^{\prime}, \beta^{\prime}>-1,
$$

where both $\tilde{F}$ and $\tilde{G}$ are real for real $x$ and analytic over a region containing $[a, b]$. Our results, in the form of theorems, treat only intervals in which the derivative $G^{\prime}$ of the oscillator function $G$ is nonnegative in (a,b] and in which any singular behavior occurs only at the left end, $x=a$. We note however, that integrals whose integrand functions satisfy (1.2) can be partitioned and transformed into a sum of integrals satisfying the latter milder conditions. We shall refer to the special case of $I_{0}$ in (1.1) in which $G(x)$ is constant as a Fourier coefficient (integral). The thrust of this approach is to reduce the more general oscillatory integral to one of this form for which an asymptotic expansion may already be

${ }^{*}$ The authors were supported by the Office of Advanced Scientific Computing Research, Office of Science, U.S. Department of Energy, under Contract DE-AC02-06CH11357. 
available. This approach has been used by one of the authors in a much more limited context (constant $F$ and no algebraic singularities). See Lyness (2008).

The asymptotic expansions of Fourier integrals exploited in this theory are assembled in Section 2 where we provide three forms of a Fourier coefficient asymptotic expansion appropriate to treat the various integrands encountered. The third form, needed when both $F$ and $G$ have algebraic singularities, appears to be new. The other forms are specializations of this and suffice for the earlier and simpler cases.

In Section 3, we introduce the general method by treating the familiar case in which both $F$ and $G$ are analytic in $[a, b]$ and $G^{\prime}(x)$ is bounded away from zero in $[a, b]$. In Section 4 we allow $G$ to include an algebraic singularity at an end point. This part of the theory applies also to an analytic minimum of $G$. In Section 5, we treat the case in which both $F$ and $G$ have algebraic singularities. Our method involves exploiting the inverse functions (that have a different structure in the different cases), in order to reduce this integral to a Fourier coefficient integral (one in which $G(x)$ is constant). Then, as mentioned above, we apply an asymptotic expansion for the Fourier coefficient integral.

In Sections 3, 4, and 5 we have introduced inverse functions in an ad hoc manner, determining early coefficients as needed, by elementary means. The intention is to enable the reader to understand precisely why they are needed and how, in extremis, they can be calculated. In Section 6 we provide a deeper theory, based on integer partitions and Faà di Bruno's celebrated result; we show how this theory can be applied to obtain both known expansions and some new ones needed in this paper.

Research in this field has been dominated by techniques based on stationary phase and steepest descent. See Olver (1974), Huybrechs and Vandewalle (2006) . Many more sophisticated expansions (Iserles and Norsett 2004) have been developed from pioneer work of Filon (1928). These require moments

$$
\mu_{j}=\int_{a}^{b} x^{j} \exp [i k G(x)] d x
$$

may involve function values within $[a, b]$ and are more powerful than the ones presented here; many are valid for all $k$.

We derive here only basic asymptotic expansions, which do not require moment values and consequently are not immediately useful for small values of $k$. However, cases in which $G(x)$ has critical points (where its derivative is zero, or has an algebraic singularity) are naturally included in the theory, which makes no appeal to stationary phase or steepest descent.

\section{Fourier Coefficient Asymptotic Expansions}

A mid-nineteenth century result, used by Poisson in his proof of the Euler-Maclaurin summation formula (also an asymptotic expansion), is now known as the Fourier coefficient asymptotic expansion (FCAE). This is presented in modern terms as a theorem. 
Theorem 2.1 (FCAE). For all positive integers $p$, and $k>0$, when $\phi(x) \in C^{(p)}[a, b]$

$$
\begin{aligned}
\int_{a}^{b} \phi(x) e^{i k x} d x= & -e^{i k b}\left\{\frac{i}{k} \phi(b)+\frac{i^{2}}{k^{2}} \phi^{\prime}(b)+\cdots+\frac{i^{p}}{k^{p}} \phi^{(p-1)}(b)\right\} \\
& +e^{i k a}\left\{\frac{i}{k} \phi(a)+\frac{i^{2}}{k^{2}} \phi^{\prime}(a)+\cdots+\frac{i^{p}}{k^{p}} \phi^{(p-1)}(a)\right\} \\
& +\frac{i^{p}}{k^{p}} \int_{a}^{b} \phi^{(p)}(x) e^{i k x} d x .
\end{aligned}
$$

This is readily established; using integration by parts, we find

$$
\int_{a}^{b} \phi(x) \exp [i k x] d x=\frac{e^{i k b} \phi(b)-e^{i k a} \phi(a)}{i k}-\frac{1}{i k} \int_{a}^{b} \phi^{\prime}(x) \exp [i k x] d x ;
$$

iterating this relation leads directly to (2.4).

By hypothesis, this theorem is limited to functions $\phi(x)$, which have a moderate degree of continuity. More sophisticated functions $\phi$ have different expansions. In this paper we need and use theorems that treat functions $\phi$, which may have algebraic singularities at one or both ends but are otherwise analytic. We shall treat in Theorem 7.1 a function that has singular behavior at $x=a$ of the form

$$
\phi(x) \sim \sum_{j=1} \phi_{j}(x-a)^{\sigma_{j}-1} ; \quad 0<\sigma_{1}<\sigma_{2} \ldots
$$

Under several conditions listed in the hypotheses of the theorem, we establish

$$
\int_{a}^{b} \phi(x) e^{i k x} d x \sim-e^{i k b} \sum_{q=0}^{p-1} \phi^{(q)}(b)\left(\frac{i}{k}\right)^{q+1}+e^{i k a} \sum_{j=1} \phi_{j} \Gamma\left(\sigma_{j}\right)\left(\frac{i}{k}\right)^{\sigma_{j}} .
$$

Section 7 is devoted to a detailed statement and a lengthy proof of the theorem.

In most of this paper, we require only a special case of the theorem, namely one restricted to functions $\phi$ having a standard algebraic singularity, (that is, one in which $\sigma_{j}=\alpha+j$ in (2.6)). This case is covered by the following theorem.

Theorem 2.2. When $\phi(x)=(x-a)^{\alpha} \psi(x)$ and $\psi(x)$ is analytic in a region containing the interval $[a, b]$, then for all positive integers $p_{1}$ and $p_{2}$

$$
\begin{aligned}
\int_{a}^{b} \phi(x) e^{i k x} d x \sim & -e^{i k b} \sum_{q=0}^{p_{1}-1} \phi^{(q)}(b)\left(\frac{i}{k}\right)^{q+1} \\
& +e^{i k a} \sum_{q=0}^{p_{2}-1} \frac{\psi^{(q)}(a)(q+\alpha) !}{q !}\left(\frac{i}{k}\right)^{q+\alpha+1} \quad \text { as } \quad k \rightarrow \infty .
\end{aligned}
$$

This expansion is itself a special case of an asymptotic expansion for the Fourier coefficient of a function

$$
f(x)=(x-a)^{\alpha}(b-x)^{\beta} r(x) \quad \alpha, \beta>-1,
$$


where $r(x)$ is analytic in a region containing $[a, b]$, which is proved, using contour integration, in Lyness (1971). Earlier proofs known to the author are by Erdelyi (1955), using neutralizer functions and general integration by parts, and by Lighthill (1958), using generalized function theory. Erdelyi's proof requires only that $r(x)$ is $C^{\left(p_{j}\right)}[a, b]$ and need not be analytic. This is discussed in Lyness (1971).

\section{General Approach}

Our general approach can be summed up in the next (two-sentence) paragraph.

We make a substitution $x=H(t)$ in the integral (1.1), $H(t)$ being chosen so that $G(H(t))=t$, thus replacing $I_{0}$ by an integral $I_{01}$ as follows:

$$
I_{0}=\int_{a}^{b} F(x) \exp [i k G(x)] d x=I_{01}:=\int_{A}^{B} \Phi(t) \exp [i k t] d t,
$$

with

$$
\Phi(t)=F(H(t)) H^{\prime}(t) ; \quad B=G(b) ; \quad A=G(a) .
$$

$I_{01}$ is a Fourier coefficient, for which an asymptotic expansion such as (2.4), (2.8), or (2.7) may be available.

However, either step described here may be infeasible or impossible. First, we must satisfy ourselves that such a function $H(t)$ exists in $[A, B]$; otherwise the substitution cannot be used. In fact, it exists whenever $G^{\prime}(x) \geq 0 \quad \forall \quad x \in[a, b]$. $H$ is known as the inverse function of $G$. The underlying theory of inverse functions is not simple; parts of the theory are deferred to Section 6 .

Second, it might seem at first sight that we need a functional form of $H(t)$ in order to construct $\Phi(t)$. Certainly, it is convenient to have such a form available. However, a glance at the asymptotic expansions for the Fourier coefficients shows that the coefficients in the expansion depend only on the behavior of the component functions at the end points of the integration interval. This property is retained by the asymptotic expansions for the oscillatory integrals. Thus it appears that we shall require only the coefficients in power expansions of $F$ and $G$ at both interval end points in order to compute the coefficients. The bulk of this paper is about how to handle this problem.

As a simple example of the power of this approach, we obtain a relatively well-known asymptotic expansion, merely by applying the FCAE expansion (2.4) to $I_{01}$ in (3.1) above. This is valid only when $G^{\prime}(x)$ is strictly positive in $[a, b]$. In this case, it is almost self-evident that the inverse function $H(t)$ exists and $\Phi(t)=F(H(t)) H^{\prime}(t)$ is analytic in $[A, B]$, so we need only apply Theorem 2.1 to the integral $I_{01}$ in (3.1) to obtain the following theorem. 
Theorem 3.1. For all positive integer $p$, and $k>0$, when $G^{\prime}(x)>0 \forall x \in[a, b]$ and $G(x), F(x)$ are analytic in a region containing $[a, b]$,

$$
\begin{aligned}
\int_{a}^{b} F(x) \exp [i k G(x)] d x=I_{01}= & -e^{i k B} \sum_{j=1}^{p} \Phi^{(j-1)}(B)\left(\frac{i}{k}\right)^{j}+e^{i k A} \sum_{j=1}^{p} \Phi^{(j-1)}(A)\left(\frac{i}{k}\right)^{j} \\
& +\left(\frac{i}{k}\right)^{p} \int_{A}^{B} \Phi^{(p)}(t) e^{i k t} d t
\end{aligned}
$$

where $\Phi, A, B$ are given by (3.2) and $H$ is the inverse function of $G$, that is, $G(H(t))=t$.

Various elementary points stand out.

First, this is of precisely the same form as the FCAE in (2.4) above. This formula simply replaces $a, b, \phi$ by $A, B, \Phi$ respectively. As in the FCAE, we note that the coefficients are local in character. Thus the $j$-th term in the first summation depends on the $j$-th derivative of $\Phi$ evaluated at $B=G(b)$. This, in turn, depends on the behavior of $H$ in a neighborhood of $t=B$ and hence on the behavior of $G$ in a neighborhood of $x=b$. Section 3.2 provides formulas based on the theory of inverse functions that express the derivatives of $\Phi$ in terms of derivatives of $f$ and $g$.

Second, we note that, when the conditions of this theorem are satisfied, one may partition the interval $(a, b)$ at some intermediate point, say $c$, and write down two distinct asymptotic expansions, one for the interval $(a, c)$ and the other for the interval $(c, b)$. However, combining these asymptotic expansions leads back to (3.3), the terms involving $C=G(c)$ disappearing. Thus, there is no immediate advantage in subdividing an interval in which $G^{\prime}(x)>0$.

In any particular application, one cannot expect $G^{\prime}(x) \geq 0$ throughout the interval $[a, b]$. However, usually the the integration interval may be subdivided into a finite number of sections in each of which either $G^{\prime}(x) \geq 0$ or $G^{\prime}(x)$ is constant or $G^{\prime}(x) \leq 0$. In this case we can partition the general interval and treat each section separately. Further subdivisions are necessary at points where either $F(x)$ or $G(x)$ have low-order derivative singularities. These partitions are mandatory.

The theorems in this paper treat cases in which singular or nonstandard behavior of $F$ or of $G$ occurs only at the left end of the interval and in which $G^{\prime}(x) \geq 0$ in the interval. At the right end of the interval, both $F$ and $G$ are locally analytic and $G^{\prime}>0$. However, by means of one or more of the identities

$\int_{a}^{b} F(x) \exp [i k G(x)] d x=\int_{a}^{b} F(a+b-y) \exp [i k G(a+b-y)] d x=\int_{a}^{b} F(x) \exp [(i(-k)(-G(x)] d x$,

one can re-express theorems so that singularities occur at the right end and, if necessary, so that $G^{\prime}(x) \leq 0$ in the integration interval. 


\subsection{Inverse Functions}

To apply the asymptotic expansion in Theorem 3.1, we need to evaluate derivatives of $\Phi(t)=F(H(t)) H^{\prime}(t)$ at $t=A=G(a)$ and at $t=B=G(b)$. To this end we reintroduce $H$ the inverse function of $G$, defined by

$$
H(t)=x \quad \text { when } \quad t=G(x) .
$$

Clearly $G$ is the inverse function of $H$. Elementary properties of this pair include

$$
H(G(x))=x, \quad G(H(t))=t, \quad H^{\prime}(t) G^{\prime}(H(t))=1,
$$

valid whenever these expressions are defined. In particular, the third relation confirms the simple nature of (3.4); the plot of $t$ as a function of $x$ is simply a reflection about the line $x=t$ of the plot of $x$ as a function of $t$.

We are particularly interested in the nature of $H$ and of $\Phi$ in the neighborhoods of the interval end points $A$ and $B$. It is convenient to treat displaced versions $g, h, f$, and $\phi$ as follows:

$g(x-a)=G(x)-G(a) ; \quad h(t-A)=H(t)-H(A) ; \quad f(x-a)=F(x) ; \quad \phi(t-A)=\Phi(t)$.

A straightforward formal manipulation shows that $g$ and $h$ are inverse functions, and that $\phi(t)=f(h(t)) h^{\prime}(t)$.

Inverse functions have been intensively studied. Fundamental results due to Lagrange form the basis of Bürmann's theorem, a short form of which is as follows.

Theorem 3.2. Let $g(z)$ be analytic in a neighborhood of $z=0$ and let $g(0)=0$. The necessary and sufficient condition that $g(z)=w$ should have a unique solution $z=h(w)$, analytic in a neighborhood of the origin, is that $g^{\prime}(0) \neq 0$.

The proof is difficult. A proof due to Landau (1904) is given in Copson (1935) pp. 121125. A full form of this theorem, which includes a quantitative relation between $g$ and $h$, is quoted in Section 6 below.

Some questions about inverse functions can be resolved by using the following elementary corollary of Theorem 3.2.

Theorem 3.3. When the power series $t=g(x)=\sum_{j=1} g_{j} x^{j}$ is absolutely convergent in a neighborhood of the origin, and $g_{1} \neq 0$, then the inverse series $x=h(t)=\sum_{j=1} h_{j} t^{j}$ exists, is unique, and is absolutely convergent in a neighborhood of the origin; and $h_{1} \neq 0$.

The proof (not given here) and the result depend critically on the circumstance that $g_{1} \neq 0$. 


\subsection{Inverse Series for $g_{1}>0$}

We now proceed with the simple case in which $G^{\prime}(x)>0$ in $[a, b]$. The hypotheses of Theorem 3.1 are valid. To apply the asymptotic expansion, we need to evaluate the early derivatives of $\Phi(t)$ at $A$ and at $B$ in terms of corresponding information about $G(x)$ and $F(x)$. As mentioned previously, even for very straightforward $g(x)$, it is rarely feasible to obtain a functional form for $h(t)$ and for $\phi(t)$. However, one can exploit the power series for $g$ about $x=0$ to obtain a power series for $h$ about $t=0$. To fix ideas, we give some early steps in this process in detail. Comparing powers of $t$ in $t=g(h(t))$ we find

$$
h_{1}=1 / g_{1} ; h_{2}=-g_{2} / g_{1}^{3} ; h_{3}=\left(2 g_{2}^{2}-g_{1} g_{3}\right) / g_{1}^{5} ; h_{4}=\left(-5 g_{2}^{3}+5 g_{1} g_{2} g_{3}-g_{1}^{2} g_{4}\right) / g_{1}^{7} \text {. }
$$

It is elementary, but tedious, to construct early terms in the expansion

$$
f(h(t))=f_{0}+f_{1} h_{1} t+\left(f_{1} h_{2}+f_{2} h_{1}^{2}\right) t^{2}+\left(f_{1} h_{3}+2 f_{2} h_{1} h_{2}+f_{3} h_{1}^{3}\right) t^{3}+O\left(t^{4}\right) .
$$

Since $h^{\prime}(t)=\sum j h_{j} t^{j-1}$ and $\phi(t)=f(h(t)) h^{\prime}(t)$, we find the early coefficients in the power series $\phi(t)=\sum \phi_{j} t^{j}$ to be

$$
\begin{array}{lcl}
\phi_{0}= & f_{0} h_{1} & =f_{0} / g_{1}, \\
\phi_{1}= & 2 f_{0} h_{2}+f_{1} h_{1}^{2} & =-2 f_{0} g_{2} / g_{1}^{3}+f_{1} / g_{1}^{2} \\
\phi_{2}= & 3 f_{0} h_{3}+3 f_{1} h_{1} h_{2}+f_{2} h_{1}^{3} & =6 f_{0} g_{2}^{2} / g_{1}^{5}-3 f_{0} g_{3} / g_{1}^{4}-3 f_{1} g_{2} / g_{1}^{4}+f_{2} / g_{1}^{3} .
\end{array}
$$

Closed expressions (6.11) for $h_{j}$ and (6.22) for $\phi_{j}$ are derived in Section 6 .

To obtain expressions for the coefficient $\Phi^{(j)}(A)$ needed in Theorem 3.1, we require only the first $j+1$ coefficients in the power expansions of $g$ and of $f$ about $x=a$. These are used in (3.6) and (3.8) to evaluate $\phi_{j}$. Then $\Phi^{(j)}(A)$ may be replaced by $j ! \phi_{j}$. Naturally the coefficients $\Phi^{(j)}(A)$ are calculated in the same way, starting with the power expansion of $f$ and $g$ about $x=b$.

Occasionally, the function $g(x)$ can be inverted analytically. But, in general, one has to rely on expansions of the functions about the end point, as is done in the foregoing example.

\section{Oscillator $G$ has critical point; $F$ analytic}

In the preceding section we treated in some detail the straightforward case in which $G(x)$ is analytic and $G^{\prime}(x)$ is bounded away from zero in the closed integration interval. In this section we treat a more sophisticated case in which $G^{\prime}(x)$ has an algebraic singularity at $x=a$. This includes an analytic minimum of $G(x)$; but in the rest of the integration interval $G^{\prime}(x)>0$; and, as before, $F(x)$ remains analytic in a region containing the integration interval. We go directly to the scaled problem and treat a function $g(x)$ whose derivative $g^{\prime}(x)$ has an algebraic singularity of order $s$ at $x=0$ but is regular at $x=b$. 


\subsection{Inverse Function of $g(x)=x^{s} e(x)$}

Let

$$
g(x)=x^{s} e(x)=x^{s}\left(e_{1}+e_{2} x+e_{3} x^{2}+\cdots\right), \quad e_{1}>0 .
$$

When $s$ is an integer exceeding $1, g(x)$ has a horizontal tangent at $x=0$ and is analytic. (When $s=1$ this reduces to the case treated in the preceding section.) Our first task is to obtain information about the inverse function $h(t)$ of this function. The previous result cannot be applied because it is invalid when, as is the case here, $g_{1}=0$.

Lemma 4.1. Let $g(x)=x^{s} e(x)$ be given by (4.1) and $e(x)$ be analytic in a neighborhood of the origin; then $h(t)$, the inverse function of $g(x)$, has an expansion convergent in a neighborhood of the origin of the form

$$
h(t)=\lambda_{1} t^{\sigma}+\lambda_{2} t^{2 \sigma}+\lambda_{3} t^{3 \sigma}+\cdots,
$$

where $\sigma=1 / s>0$.

Proof. We are seeking $h(t)$ the inverse function $t=g(x)$; we take the $s$-th root of the relation $t=g(x)$ and expand $(e(x))^{\sigma}$ using either the binomial theorem iteratively or a multinomial theorem such as Theorem 6.2 to obtain successively

$$
\tau=: t^{\sigma}=x(e(x))^{\sigma}=: \gamma(x)=\gamma_{1} x+\gamma_{2} x^{2}+\gamma_{3} x^{3}+\cdots \quad ; \quad \gamma_{1}=e_{1}^{1 / s}>0 .
$$

Since $\tau=\gamma(x)$ satisfies all the conditions of Theorem 3.3, we may apply it to establish that the inverse function of $\gamma(x)$, which we denote by $\lambda(\tau)$, exists and is unique. Thus

$$
x=\lambda(\tau)=\lambda_{1} \tau+\lambda_{2} \tau^{2}+\lambda_{3} \tau^{3}+\cdots .
$$

If we set $\tau=t^{\sigma} \mathrm{r}$, it follows that $x=\lambda\left(t^{\sigma}\right)$. Since this expresses $x$ as a function of $t$, it is, ipso facto, an inverse function $h(t)$ of $g(x)$. That is,

$$
h(t)=\lambda_{1} t^{\sigma}+\lambda_{2} t^{2 \sigma}+\lambda_{3} t^{3 \sigma}+\cdots .
$$

Note that the relationship beween $\gamma_{i}$ and $\lambda_{i}$ is precisely the same as that between $h_{i}$ and $g_{i}$ in (3.6).

The corresponding expansion for $\phi=f(h(t)) h^{\prime}(t)$ may be obtained by inserting expression (4.5) for $h(t)$ into $f(x)=\sum_{j=0} f_{j} x^{j}$, multiplying by the corresponding expression for $h^{\prime}(t)$, and collecting together contributions to specific powers of $t$ in the usual way. We obtain the expansion of the form

$$
\phi(t)=f(h(t)) h^{\prime}(t)=t^{-1} \sum_{j=1} \phi_{j} t^{j \sigma} .
$$

The early cofficients are

$$
\phi_{1}=\sigma \lambda_{1} f_{0} ; \quad \phi_{2}=\sigma\left(2 \lambda_{2} f_{0}+\lambda_{1}^{2} f_{1}\right) ; \quad \phi_{3}=\sigma\left(3 \lambda_{3} f_{0}+3 \lambda_{1} \lambda_{2} f_{1}+\lambda_{1}^{3} f_{2}\right) .
$$

A closed expression (6.22) for these coefficients $\phi_{j}$ is given in Section 6. We are now in a position to establish the following: 
Theorem 4.1. Let $s=1 / \sigma>0$. Let

$$
g(x)=x^{s} e(x)=x^{s}\left(e_{1}+e_{2} x+e_{3} x^{2}+\cdots\right), \quad e_{1}>0 .
$$

Let $f(x), e(x)$ be analytic in a region including $[0,1]$ and $g^{\prime}(x)>0 \forall x \in(0,1]$. Then

$$
\int_{0}^{b} f(x) e^{i K g(x)} d x \sim-e^{i K b} \sum_{q=0}^{p_{1}-1} \phi^{(q)}(b)\left(\frac{i}{K}\right)^{q+1}+\sum_{j=1} \phi_{j} \Gamma(j \sigma)\left(\frac{i}{K}\right)^{j \sigma},
$$

where the coefficients $\phi_{j}$ are defined through (4.6), the expansion about $t=0$ of

$$
\phi(t)=f(h(t)) h^{\prime}(t)=\sum_{j=1} \phi_{j} t^{j \sigma-1} .
$$

Here $h(x)$ is the inverse function of $g(x)$.

Proof. We recall that a straightforward substitution gives

$$
\int_{0}^{b} f(x) \exp [i K g(x)] d x=I_{2}=\int_{0}^{b} \phi(t) \exp [i K t] d t .
$$

This is of precisely a form to which Theorem 7.1 may be applied. We set $a=0$ and $\sigma_{j}=\sigma j$ in that theorem, and we find the right-hand side of (4.9), together with a remainder term of the appropriate order; this establishes Theorem 4.1. 


$$
4.2 g(x)=x^{n / d}\left(g_{2}+g_{3} x+\cdots\right) ; \quad g_{2}>0 ; \quad f(x) \in \mathcal{A}[0,1]
$$

This subsection is devoted to some minor simplifications that occur when $s$ is rational, say, $s=n / d$, where $n$ and $d$ are integers. One reason for treating rational $s$ is that $h(t)$ and $\phi(t)$ can then be expressed as a sum of $n$ functions to each of which Theorem 2.2 may be applied. For example, when $s=3 / 2$ and so $\sigma=2 / 3$, we can reorder expansion (4.6) as

$$
\begin{aligned}
h(t) & =t^{2 / 3}\left(\lambda_{1}+\lambda_{4} t^{2}+\lambda_{7} t^{4}+\ldots\right)+t^{4 / 3}\left(\lambda_{2}+\lambda_{5} t^{2}+\lambda_{8} t^{4}+\ldots\right)+t^{2}\left(\lambda_{3}+\lambda_{6} t^{2}+\lambda_{9} t^{4}+\ldots\right) \\
\phi(t) & =t^{-1}\left\{t^{2 / 3}\left(\phi_{1}+\phi_{4} t^{2}+\phi_{7} t^{4}+\ldots\right)+t^{4 / 3}\left(\phi_{2}+\phi_{5} t^{2}+\phi_{8} t^{4}+\ldots\right)+t^{2}\left(\phi_{3}+\phi_{6} t^{2}+\phi_{9} t^{4}+\ldots\right)\right\} \\
& =t^{-1}\left\{t^{2 / 3} \Psi_{1}(t)+t^{4 / 3} \Psi_{2}(t)+t^{2} \Psi_{3}(t)\right\}=\phi_{1}(t)+\phi_{2}(t)+\phi_{3}(t) .
\end{aligned}
$$

Naturally, a corresponding reordering exists whenever $s$ is rational. When $\sigma=d / n$, we set

$$
\phi(t)=t^{-1} \sum_{j=1} \phi_{j} t^{j d / n}:=\sum_{r=1}^{n} t^{r \sigma-1} \Psi_{r}(t) ; \quad \Psi_{r}(t):=\sum_{k=0}^{\infty} \phi_{k n+r} t^{k d} \quad r=1,2, \ldots, n .
$$

$\Psi_{r}(t)$ is a subseries of a series $\sum \phi_{j} \tau^{j}$. Hence, it is also an analytic function of $t$ in a neighborhood of the origin. Its Taylor coefficients are

$$
\Psi_{r}^{(q)}(0) / q != \begin{cases}\phi_{n k+r} & \text { when } q=k d \\ 0 & \text { otherwise. }\end{cases}
$$

We have already established Theorem 4.1 for all $\sigma>0$. When $\sigma$ is rational, however, a proof of the same theorem becomes simpler. Since

$\int_{0}^{b} f(x) \exp [i K g(x)] d x=I_{2}=\int_{0}^{b} \phi(t) \exp [i K t] d t=\sum_{r=1}^{n} \int_{0}^{b} t^{r \sigma-1} \Psi_{r}(t) \exp [i K t] d t=\sum_{r=1}^{n} J_{r}$,

we simply employ Theorem 2.2 to provide an asymptotic expansion for $J_{r}$. Introducing the values of the derivatives given by (4.14) into the individual expansions, summing over $r$, and reordering the summation back to its original form, we recover Theorem 4.1.

Note that this proof involves little more than series manipulation. The steps leading to (4.15) involve only the elementary algebra required to reorder a series. Theorem 2.2 is then applied to each integral $J_{r}$. Finally, the steps to establishing the theorem simply involve putting the series back in its original order, using elementary algebra. 


\section{Both $F$ and $G$ have algebraic singularities}

In this section, we briefly treat a situation in which both $F(x)$ and $G(x)$ have a standard algebraic singularity at $x=a$.

Theorem 5.1. Let $\alpha>0$ and $s=1 / \sigma>0$. Let

$$
\begin{array}{lll}
g(x)=x^{s} e(x)=x^{s}\left(e_{1}+e_{2} x+e_{3} x^{2}+\cdots\right) ; & e_{1}>0 \\
f(x)=x^{\alpha} \tilde{f}(x)=x^{\alpha}\left(f_{0}+f_{1} x+f_{2} x^{2}+\cdots\right) ; & f_{0}>0 .
\end{array}
$$

Let $\tilde{f}(x), e(x)$ be analytic in a region including $[0,1]$ and $g^{\prime}(x)>0 \forall x \in(0,1]$. Then

$$
\int_{0}^{b} f(x) e^{i K g(x)} d x \sim-e^{i K b} \sum_{q=0}^{p_{1}-1} \phi^{(q)}(b)\left(\frac{i}{K}\right)^{q+1}+\sum_{j=1} \phi_{j} \Gamma((j+\alpha) \sigma)\left(\frac{i}{K}\right)^{(j+\alpha) \sigma},
$$

where the coefficients $\phi_{j}$ are defined through the expansion about $t=0$ of

$$
\phi(t)=f(h(t)) h^{\prime}(t)=t^{\sigma \alpha-1} \sum_{j=1} \phi_{j} t^{j \sigma-1} .
$$

Here $h(x)$ is the inverse function of $g(x)$.

Since $g(x)=x^{1 / \sigma} e(x)$ is the same as in the preceding section, its inverse function $h(t)$ is of the form described in Lemma 4.1. We have

$$
h(t)=\sum_{j=1} \lambda_{j} t^{j \sigma} ; \quad h^{\prime}(t)=t^{-1} \sigma \sum_{j=1} j \lambda_{j} t^{j \sigma} .
$$

We shall require the corresponding expansion of $h^{\alpha}$. Using a mutinomial expansion gives

$$
(h(t))^{\alpha}=\left(\lambda_{1} t^{\sigma}\right)^{\alpha}\left(1+\frac{\alpha \lambda_{2}}{\lambda_{1}} t^{\sigma}+\ldots\right) .
$$

As usual, we are interested in

$$
\int_{0}^{b} f(x) \exp [i K g(x)] d x=I_{2}=\int_{0}^{b} \phi(t) \exp [i K t] d t \quad \text { with } \quad \phi(t)=f(h(t)) h^{\prime}(t) .
$$

so we look for the coefficients in a power expansion of

$$
\phi(t)=f(h(t)) h^{\prime}(t)=(h(t))^{\alpha} h^{\prime}(t)\left(f_{0}+f_{1} h(t)+f_{2}(h(t))^{2}+\cdots\right)
$$

In the by-now-familiar process of replacing $h^{\alpha}, h$, and $h^{\prime}$ by their expansions in (5.5) and (5.4), we find

$$
\phi(t)=f(h(t)) h^{\prime}(t)=t^{\sigma \alpha} \sum_{j=1} \phi_{j} t^{j \sigma-1} .
$$

the earlier coefficients being

$$
\phi_{1}=\sigma \lambda_{1}^{\alpha} f_{0} \lambda_{1} ; \quad \phi_{2}=\sigma \lambda_{1}^{\alpha}\left(f_{0}(\alpha+2) \lambda_{2}+f_{1} \lambda_{1}^{2}\right) .
$$

A closed expression (6.22) for these coefficients $\phi_{j}$ is given in Section 6. We now note that $\phi(t)$ is of a form to which Theorem 7.1 may be applied. We set $a=0$ and $\sigma_{j}=(j+\alpha) \sigma$ in that theorem, and we find the right-hand side of (5.2), together with a remainder term of the appropriate order; this establishes Theorem 5.1. 


\section{Closed Forms}

\subsection{Notation and Background}

\subsubsection{Multi-Index Notation for Integer Partitions}

Let $\Pi(n) \subseteq \mathbb{N}_{0}^{n}$ be defined by

$$
\pi \in \Pi(n) \quad \text { iff } \quad \pi_{1}+2 \pi_{2}+3 \pi_{3}+\cdots+n \pi_{n}=n .
$$

We identify $\Pi(n)$ as the set of all partitions of the integer $n$. Multi-index $\pi$ corresponds to the partition with $\pi_{1} 1$ 's, $\pi_{2} 2$ 's, and so forth. The number of addends in the partition is the absolute value of the multi-index,

$$
|\pi|=\pi_{1}+\pi_{2}+\cdots+\pi_{n} .
$$

We define the multinomial coefficient as

$$
\left(\begin{array}{c}
z \\
\pi
\end{array}\right)=\frac{z !}{(z-|\pi|) ! \pi_{1} ! \pi_{2} ! \cdots \pi_{n} !}=\frac{z(z-1) \cdots(z-|\pi|+1)}{\pi_{1} ! \pi_{2} ! \cdots \pi_{n} !},
$$

which is entire in $z$. This notation is unconvential; only when $z=|\pi|$ does the convential multinomial coefficient agree with (6.3). Other authors have also used the notation (6.3), which has the advantage of reducing to the standard binomial coefficient when $n=1$.

\subsubsection{Faà di Bruno's Formula}

The formula for high-order derivatives of a composition of functions, known as Faà di Bruno's formula, has a rich history. (Johnson (2002)). We state a version here using the multi-index notation just presented.

Theorem 6.1 (Faà di Bruno's Formula). When functions $f$ and h have sufficiently many derivatives,

$$
\frac{1}{n !} \frac{d^{n}}{d x^{n}} f(h(x))=\sum_{\pi \in \Pi(n)}\left(\begin{array}{c}
|\pi| \\
\pi
\end{array}\right) \frac{f^{(|\pi|)}(h(x))}{|\pi| !} \prod_{i=1}^{n}\left[\frac{h^{(i)}(x)}{i !}\right]^{\pi_{i}} .
$$

\subsubsection{Infinite Multinomial Theorem}

An immediate consequence of Faà di Bruno's formula is obtained by taking $f(y)=y^{r}$. When $h(x)$ has sufficient derivatives at $x=0$, and $h\left(x_{0}\right) \neq 0$ or $n \leq r$,

$$
\left.\frac{1}{n !} \frac{d^{n}}{d x^{n}}[h(x)]^{r}\right|_{x=x_{0}}=\sum_{\pi \in \Pi(n)}\left(\begin{array}{l}
r \\
\pi
\end{array}\right)\left[h\left(x_{0}\right)\right]^{r-|\pi|} \prod_{i=1}^{n}\left[\frac{h^{(i)}\left(x_{0}\right)}{i !}\right]^{\pi_{i}} .
$$

When $h(x)$ is expanded as a power series, this provides the following classical result.

Theorem 6.2 (Infinite Multinomial Theorem). When $a_{0} \neq 0$ and both series converge,

$$
\left(a_{0}+a_{1} x+a_{2} x^{2}+\cdots\right)^{r}=\sum_{n=0}^{\infty}\left[\sum_{\pi \in \Pi(n)}\left(\begin{array}{l}
r \\
\pi
\end{array}\right) a_{0}^{r-|\pi|} \prod_{i=1}^{n} a_{i}^{\pi_{i}}\right] x^{n} .
$$


Note that setting $a_{n}=0$ for $n>1$ in (6.6) gives the conventional binomial theorem

$$
\left(a_{0}+a_{1} x\right)^{r}=\sum_{n=0}^{\infty}\left(\begin{array}{l}
r \\
n
\end{array}\right) a_{0}^{r-n} a_{1}^{n} x^{n} .
$$

\subsubsection{Lagrange-Bürmann Series}

The following is a consequence of Bürmann's theorem (1799), which is a generalization of a result by Lagrange (1768). See Whittaker \& Watson (1953). The case in which $g^{\prime}\left(x_{0}\right)=0$ is treated by Hurwitz and Courant (1925).

Theorem 6.3 (Lagrange-Bürmann Series). Let functions $f(x), g(x)$ be analytic in a neighborhood of $x=x_{0}$ with $g^{\prime}\left(x_{0}\right) \neq 0$; let $h(t)$ be the inverse function of $g$. The invers function, and also the composition $f(h(t))$ are single-valued and analytic in a neighborhood of $t=g\left(x_{0}\right)$. The $n$-th derivative of the composition at $t=g\left(x_{0}\right)$, with $n \geq 1$, is

$$
\left.\frac{d^{n}}{d t^{n}} f(h(t))\right|_{t=g\left(x_{0}\right)}=\left.\frac{d^{n-1}}{d x^{n-1}}\left[f^{\prime}(x)\left(\frac{x-x_{0}}{g(x)-g\left(x_{0}\right)}\right)^{n}\right]\right|_{x=x_{0}} .
$$




\subsection{New Results}

\subsubsection{Power Series Reversion}

Theorem 6.4. Let the function $g(x)$ be given by the series, convergent in a neighborhood of $x=h_{1}$,

$$
g(x)=A+g_{1}(x-a)^{s r}+g_{2}(x-a)^{(1+s) r}+g_{3}(x-a)^{(2+s) r}+\cdots,
$$

where $g_{1} \neq 0$, Re $s>0$, and $\operatorname{Re} r>0$. Let $\sigma=1 / s$ and $\rho=1 / r$. Then $g(x)$ has an inverse function,

$$
h(t)=a+h_{1}(t-A)^{\rho \sigma}+h_{2}(t-A)^{(1+\rho) \sigma}+h_{3}(t-A)^{(2+\rho) \sigma}+\cdots,
$$

where $\sigma=1 / s, \rho=1 / r$, and setting $\sigma_{n}=(n+\rho) \sigma$

$$
h_{1}=g_{1}^{-\sigma_{0}} ; \quad h_{n+1}=\frac{\rho}{\sigma} \sigma_{n} \sum_{\pi \in \Pi(n)}\left(\begin{array}{c}
-\sigma_{n} \\
\pi
\end{array}\right) g_{1}^{-\sigma_{n}-|\pi|} \prod_{i=1}^{n} g_{i+1}^{\pi_{i}} .
$$

The inverse is valid, in the sense that $g(h(t))=t$, when $t$ is in some neighborhood of $t=A$, and $t$ is in the image of the function $g(x)$.

Proof. Let $t=g(x)$. We seek an inverse function $x=h(t)$ such that $g(h(t))=t$. To be able to apply Bürmann's theorem, we need analytic functions. Set

$$
y=(x-a)^{r}, \quad \tau=(t-A)^{\sigma} .
$$

Then we may write

$$
\begin{aligned}
\gamma(y) & =\tau=y[\tilde{g}(y)]^{\sigma}, \quad \tilde{g}(y)=g_{1}+g_{2} y+g_{3} y^{2}+\cdots ; \\
x & =a+\tau^{\rho} f(y), \quad f(y)=\tilde{g}(y)^{-\rho \sigma}=\left(\frac{y}{\tau}\right)^{\rho} .
\end{aligned}
$$

Because $\tilde{g}(0)=g_{1} \neq 0$, we see that the introduced functions $f(y)$ and $\gamma(y)=\tau$ are analytic in a neighborhood of $y=0$, and $\gamma^{\prime}(0) \neq 0$. Therefore, Bürmann's theorem and equation (6.8) apply:

$$
\left.\frac{d^{n}}{d \tau^{n}} f(y)\right|_{\tau=0}=\left.\frac{d^{n-1}}{d y^{n-1}}\left[f^{\prime}(y)\left(\frac{y}{\tau}\right)^{n}\right]\right|_{y=0} .
$$

We may substitute the definition of $f(y)$ to obtain

$$
\left(\frac{y}{\tau}\right)^{n} f^{\prime}(y)=\left(\frac{y}{\tau}\right)^{n} \frac{d}{d y}\left(\frac{y}{\tau}\right)^{\rho}=\frac{\rho}{n+\rho} \frac{d}{d y}\left(\frac{y}{\tau}\right)^{n+\rho} .
$$

Hence, defining $h_{n+1}$ as the $n$-th Taylor series coefficient of $f(y)$, we have

$$
h_{n+1}=\left.\frac{1}{n !} \frac{d^{n}}{d \tau^{n}} f(y)\right|_{\tau=0}=\left.\frac{\rho}{n+\rho} \frac{1}{n !} \frac{d^{n}}{d y^{n}}\left(\frac{y}{\tau}\right)^{n+\rho}\right|_{y=0} .
$$


Noting $y / \tau=[\tilde{g}(y)]^{-\sigma}$, we may apply the infinite multinomial result (6.5),

$$
\left.\frac{1}{n !} \frac{d^{n}}{d y^{n}}[\tilde{g}(y)]^{-\sigma_{n}}\right|_{y=0}=\sum_{\pi \in \Pi(n)}\left(\begin{array}{c}
-\sigma_{n} \\
\pi
\end{array}\right) g_{1}^{-\sigma_{n}-|\pi|} \prod_{i=1}^{n} g_{i+1}^{\pi_{i}} .
$$

This establishes (6.11) a closed expression for the coefficient $h_{n+1}$. To obtain the series (6.10) one need only expand $f(y)$ as a Taylor series about $\tau=0$ in (6.14) and substitute $\tau=(t-A)^{\sigma}$.

\subsubsection{Expansion of $\phi(t)=f(h(t)) h^{\prime}(t)$}

Theorem 6.5. Let the functions $f(x)$ and $g(x)$ be defined by the series, convergent in a neighborhood of $x=a$,

$$
\begin{array}{ll}
f(x)=(x-a)^{\alpha-1} \tilde{f}(x-a) ; \quad \tilde{f}(y) & =f_{0}+f_{1} y+f_{2} y^{2}+\cdots, \\
g(x)=A+(x-a)^{s} \tilde{g}(x-a) ; \quad \tilde{g}(y) & =g_{1}+g_{2} y+g_{3} y^{2}+\cdots,
\end{array}
$$

where $\operatorname{Re} s>0$ and $\tilde{f}(y)$ and $\tilde{g}(y)$ are functions with convergent power series in a neighborhood of $y=0$, If $g_{1} \neq 0$, then an inverse function $h(t)$ satisfying $g(h(t))=t$ exists and

$$
\phi(t)=f(h(t)) h^{\prime}(t)=\phi_{0}(t-A)^{\alpha \sigma-1}+\phi_{1}(t-A)^{(1+\alpha) \sigma-1}+\cdots,
$$

where $\sigma=1 / s, \rho=1 / r$, and $\sigma_{n}=(n+\rho+\alpha) \sigma$ :

$$
\phi_{n}=\rho \sigma \sum_{k=0}^{n} f_{n-k} \sum_{\pi \in \Pi(k)}\left(\begin{array}{c}
-\sigma_{n} \\
\pi
\end{array}\right) g_{1}^{-\sigma_{n}-|\pi|} \prod_{i=1}^{k} g_{i+1}^{\pi_{i}} .
$$

Proof. As in the proof of the previous theorem, we let $t=g(x)$ and then seek analytic functions to which we may usefully apply Bürmann's theorem. Here we set

$$
y=x-a, \quad \tau=(t-A)^{\sigma},
$$

and introduce

$$
\gamma(y)=\tau=y[\tilde{g}(y)]^{\sigma}, \quad F^{\prime}(y)=\tilde{f}(y)[\tilde{g}(y)]^{-(\alpha-1) \sigma}=\frac{f(x)}{\tau^{\alpha-1}} .
$$

We take $F(y)$ to be any antiderivative of $F^{\prime}(y)$ just defined; because $\tilde{g}(0)=g_{1} \neq 0$, both $F(y)$ and $\gamma(y)$ are analytic in a neighborhood of $y=0$. Additionaly, $\gamma^{\prime}(y) \neq 0$, so that Bürmann's theorem may be applied to the pair. First, we show how doing so is useful. Using the chain rule and the formula $d \tau / d t=\sigma \tau^{1-s}$, we establish

$$
\phi(t)=f(h(t)) h^{\prime}(t)=\tau^{\alpha-1} F^{\prime}(y) \frac{d y}{d t}=\sigma \tau^{\alpha-s} \frac{d}{d \tau} F(y) .
$$

Expanding $d F(y) / d \tau$ as a Taylor series in $\tau$ yields (6.21) with

$$
\phi_{n}=\left.\frac{\rho \sigma}{n !} \frac{d^{n+1}}{d \tau^{n+1}} F(y)\right|_{\tau=0} .
$$


Now we apply Bürmann's theorem and equation (6.8):

$$
\left.\frac{d^{n+1}}{d \tau^{n+1}} F(y)\right|_{\tau=0}=\left.\frac{d^{n}}{d y^{n}}\left[F^{\prime}(y)\left(\frac{y}{\tau}\right)^{n+1}\right]\right|_{y=0}=\left.\frac{d^{n}}{d y^{n}} \frac{\tilde{f}(y)}{[\tilde{g}(y)]^{(n+\alpha) \sigma}}\right|_{y=0} .
$$

We expand the last expression using the Leibniz (product) rule:

$$
\phi_{n}=\left.\frac{\sigma}{n !} \frac{d^{n}}{d \tau^{n}} F(y)\right|_{\tau=0}=\left.\sigma \sum_{k=0}^{n} \frac{\tilde{f}^{(n-k)}(0)}{(n-k) !} \frac{1}{k !} \frac{d^{k}}{d y^{k}}[\tilde{g}(y)]^{-(n+\alpha) \sigma}\right|_{y=0} .
$$

We then apply the infinite multinomial result (6.5),

$$
\left.\frac{1}{k !} \frac{d^{k}}{d y^{k}}[\tilde{g}(y)]^{-\sigma_{n}}\right|_{y=0}=\sum_{\pi \in \Pi(k)}\left(\begin{array}{c}
-\sigma_{n} \\
\pi
\end{array}\right) g_{1}^{-\sigma_{n}-|\pi|} \prod_{i=1}^{k} g_{i+1}^{\pi_{i}} .
$$




\section{An Umbrella Fourier Coefficient Asymptotic Expansion}

In Section 3, we assembled several asymptotic expansions for Fourier coefficient-type integrals required in this paper. In this section, we give the proof of the umbrella theorem, briefly mentioned there, which includes the others as special cases.

This section is a stand-alone section, requiring no input from the rest of the paper. It is devoted exclusively to establishing the following theorem.

Theorem 7.1. Let $s_{n}(x)$ be the first $n$ terms of a generalized power series representation of $\phi(x)$ about $x=a$, with remainder $r_{n}(x)$,

$$
s_{n}(x)=\sum_{j=1}^{n} \phi_{j}(x-a)^{\sigma_{j}-1}, \quad r_{n}(x)=\phi(x)-s_{n}(x),
$$

where the exponents satisfy $0<\operatorname{Re} \sigma_{1} \leq \operatorname{Re} \sigma_{2} \leq \cdots$. Given any nonnegative integer $p$, let $n$ be the smallest integer such that $r_{n}(x) \in C^{(p-1)}[a, b], r_{n}^{(p)}(x) \in L^{2}[a, b]$, and $r_{n}^{(q)}(a)=0$ for each $q<p$; assume such $n$ always exists for the given series. Then, for $k \neq 0$, provided the integral exists,

$$
\int_{a}^{b} \phi(x) e^{i k x} d x=-e^{i k b} \sum_{q=0}^{p-1} \phi^{(q)}(b)\left(\frac{i}{k}\right)^{q+1}+e^{i k a} \sum_{j=1}^{n} \phi_{j} \Gamma\left(\sigma_{j}\right)\left(\frac{i}{k}\right)^{\sigma_{j}}+\left(\frac{i}{k}\right)^{p} R_{p},
$$

where the remainder is

$$
R_{p}=-\int_{b}^{a+i \infty / k} s_{n}^{(p)}(x) e^{i k x} d x+\int_{a}^{b} r_{n}^{(p)}(x) e^{i k x} d x .
$$

Moreover, (7.2) constitutes an asymptotic expansion valid for $|k| \rightarrow \infty, \operatorname{Im} k \geq 0$.

Proof. Split $\phi$ into the partial sum and remainder:

$$
\int_{a}^{b} \phi(x) e^{i k x} d x=\int_{a}^{b} s_{n}(x) e^{i k x} d x+\int_{a}^{b} r_{n}(x) e^{i k x} d x .
$$

Let $L=b-a$. The variable substitution $x=i t / k+a$ transforms the contribution to the first integral of each term of $s_{n}(x)$ into a lower incomplete gamma function:

$$
\begin{gathered}
\int_{a}^{b} \phi_{j}(x-a)^{\sigma_{j}-1} e^{i k x} d x=e^{i k a} \phi_{j}\left(\frac{i}{k}\right)^{\sigma_{j}} \int_{0}^{-i k L} t^{\sigma_{j}-1} e^{-t} d t \\
\int_{0}^{-i k L} t^{\sigma_{j}-1} e^{-t} d t=\gamma\left(\sigma_{j},-i k L\right)=\Gamma\left(\sigma_{j}\right)-\Gamma\left(\sigma_{j},-i k L\right) \\
=\Gamma\left(\sigma_{j}\right)-\int_{-i k L}^{+\infty} t^{\sigma_{j}-1} e^{-t} d t .
\end{gathered}
$$

The branch cut for $t^{\sigma_{j}-1}$ and the contour for the final integral are chosen so they do not intersect and so that they are consistent with the definition of the upper incomplete gamma function. Moreover, we take the branch cut and contour to be the same for each $j$, so that 
no problems arise when we sum. The upper limit denoted by $+\infty$ stands for real $t$ in the limit as $t \rightarrow+\infty$. We transform this integral back using $x=i t / k+a$ :

$$
\int_{a}^{b} \phi_{j}(x-a)^{\sigma_{j}-1} e^{i k x} d x=e^{i k a} \phi_{j} \Gamma\left(\sigma_{j}\right)\left(\frac{i}{k}\right)^{\sigma_{j}}-\int_{b}^{a+i \infty / k} \phi_{j}(x-a)^{\sigma_{j}-1} e^{i k x} d x .
$$

The integral limit denoted by $a+i \infty / k$ stands for $a+i t / k$ in the limit as the real $t \rightarrow+\infty$. Summing over $n$ terms, we find

$$
\int_{a}^{b} s_{n}(x) e^{i k x} d x=e^{i k a} \sum_{j=1}^{n} \phi_{j} \Gamma\left(\sigma_{j}\right)\left(\frac{i}{k}\right)^{\sigma_{j}}-\int_{b}^{a+i \infty / k} s_{n}(x) e^{i k x} d x .
$$

Substituting back into (7.4), we find

$$
\int_{a}^{b} \phi(x) e^{i k x} d x=e^{i k a} \sum_{j=1}^{n} \phi_{j} \Gamma\left(\sigma_{j}\right)\left(\frac{i}{k}\right)^{\sigma_{j}}+R_{0},
$$

recalling the definition of $R_{q}$ given by (7.3). Performing integration by parts on both integrals ocurring in $R_{q}$ leads to a recurrence formula, valid for $q<p$ :

$$
\begin{aligned}
i k R_{q} & =-\left.s_{n}^{(q)}(x) e^{i k x}\right|_{b} ^{a+i \infty / k}+\left.r_{n}^{(q)}(x) e^{i k x}\right|_{a} ^{b}-R_{q+1} \\
& =\phi^{(q)}(b) e^{i k b}-R_{q+1} .
\end{aligned}
$$

The boundary term at $x=a+i \infty / k$ vanishes because the decay of $e^{i k x}$ dominates in that limit, while the boundary term at $x=a$ vanishes because we assumed $r_{n}^{(q)}(a)=0$ for $q<p$. Applying the recurrence $p$ times, we easily establish

$$
R_{0}=-e^{i k b} \sum_{q=0}^{p-1} \phi^{(q)}(b)\left(\frac{i}{k}\right)^{q+1}+\left(\frac{i}{k}\right)^{p} R_{p}
$$

proving the equality $(7.2)$.

Next, we show that (7.2) constitutes an asymptotic expansion. This occurs if $R_{p} \rightarrow 0$ in the limit as $|k| \rightarrow \infty$ and if the lowest order of any term in the summations of (7.2) is $O\left(k^{-p}\right)$. The latter condition is satisfied provided $\operatorname{Re} \sigma_{n} \leq p$. This must be; otherwise the last term, $\phi_{n}(x-a)^{\sigma_{n}-1}$, could be omitted from the truncated series $s_{n}(x)$ and the regularity conditions imposed on $r_{n}(x)$ would still hold for $r_{n-1}(x)$. But $n$ is defined as the smallest integer for which those conditions hold.

It remains to show that $R_{p} \rightarrow 0$ as $|k| \rightarrow \infty$. We consider each integral in (7.3) separately. The function $r_{n}^{(p)}(x)$ satisfies the conditions of the Riemann-Lebesgue lemma, which states

$$
\int_{a}^{b} r_{n}^{(p)}(x) e^{i k x} d x \rightarrow 0 \quad \text { as }|k| \rightarrow \infty, \operatorname{Im} k \geq 0,
$$

exactly as we require. For the other integral, we have

$$
-\int_{b}^{a+i \infty / k} s_{n}^{(p)}(x) e^{i k x} d x=e^{i k a} \sum_{j=1}^{n} \phi_{j} \frac{\Gamma\left(\sigma_{j}\right)}{\Gamma\left(\sigma_{j}-p\right)} \Gamma\left(\sigma_{j}-p,-i k L\right)\left(\frac{i}{k}\right)^{\sigma_{j}-p} .
$$


Now, since $\Gamma(\alpha, z)=O\left(z^{\alpha-1}\right)$ as $|z| \rightarrow \infty$, Re $z \geq 0$ (see, e.g., Abromowitz \& S pp ??), it follows that

$$
-\int_{b}^{a+i \infty / k} s_{n}^{(p)}(x) e^{i k x} d x=O\left(k^{-1}\right) \quad \text { as }|k| \rightarrow \infty, \operatorname{Im} k \geq 0 .
$$

In the special case in which $\phi(x)$ is analytic in $(a, b]$ and has an algebraic singularity at $x=a$, that is

$$
\phi(x)=(x-a)^{\alpha} \psi(x) a \sim(x-a)^{\alpha} \sum_{j=1}^{\infty} \frac{\psi^{(j-1)}(a)}{(j-1) !}(x-a)^{j-1},
$$

we simply apply Theorem 7.1 with $\sigma_{j}=j+\alpha$ and $\phi_{j}=\psi^{(j-1)}(a) /(j-1$ !) to obtain Theorem 2.2. And in the very special case in which there is no singularity, that is $\alpha=0$ this reduces to Theorem 2.1 . 


\section{Concluding Remarks}

The thrust of this paper is to develop a basic method for constructing asymptotic expansions in inverse powers of $k$ for oscillatory integrals of the form $\int_{a}^{b} F(x) \exp [i k G(x)] d x$. Here, both $F$ and $G$ are real on the real line, are analytic over the open integration interval but may have algebraic singularities at either or both interval ends. The conventional approach to constructing such expansions is based on stationary phase or steepest descent. The approach described here is based on the theory of inverse functions and asymptotic expansions for finite Fourier integrals.

The principal expansions derived in this paper are stated in Theorems 3.1, 4.1, and 5.1. The first of these is wellknown. It refers to a situation in which $G^{\prime}(x)$ is bounded away from zero in the closed integration interval. It is included to provide a description of the basic approach in a straightforward setting. The second and third theorems refer to cases in which $G$ may have an algebraic singularity or have a stationary point at an interval endpoint, specifically, $G(x)=(x-a)^{s} \tilde{G}(x)$, where $\tilde{G}$ is analytic in the integration interval. In the second theorem, $F$ is analytic. In the third, $F$ also has an algebraic singularity at an end point.

The first five sections cover all the material in a straightforward manner. In Section 2 we assemble the various Fourier coefficient expansions that we need. In Sections 3, 4, and 5 we obtain the early terms in the respective expansions employing a direct approach. By the end of Section 5 the reader has been treated to an exposition of this general method; the form of the required asymptotic expansion is available; and a few of the early coefficients have been specified.

In the final third of this paper, we proceed at a deeper mathematical level. In Section 6 we provide closed-form expressions for the individual coefficients appearing in these expansions. This is part of the theory of inverse functions. These closed forms are based on Faà de Bruno's celebrated result and on Bürmann's theorem and involve finite summations over specified partitions of integers. To our knowledge, Theorems 6.4 and 6.5 are new. They both provide closed formulas for coefficients in expansions of the inverse function $h(t)$ and $\phi(t)$ in cases in which $g(x)$ and $f(x)$ have nonelementary behavior.

We also defer to section 7 a three page proof of a new Fourier coefficient expansion which is required to cover a large class of integrand functions. 


\section{References}

Bürmann, H. H. (1799) Memoires de l'Institut,II. (1799) p.13

Copson, E.T. (1935) Theory of Functions of a Complex Variable, Oxford University Press.

Erdelyi, A. (1955) "Asymptotic representations of Fourier integrals and the method of stationary phase" J. Soc. Indust. Appl. Math., v. 3, 1955, pp. 17-27. MR 17, 29.

Filon, J.N.G. (1928), "On a quadrature formula for trigonometric integrals," Proc. Roy. Soc. Edinburgh, v. 49, 1928, pp.38-47

Hurwitz, A. and R. Courant (1925) Algemeine Funktiontheorie, Springer, Berlin 1925

Huybrechs, D. and Vandewalle,S. (2006) "On the evaluation of highly oscillatory Integrals by analytic continuation," SIAM J. Numer. Anal., v. 44, 2006 pp. 1026-1048.

Iserles, A. and Nørsett, S. P. (2004) "On Quadrature Methods for Highly Oscillatory Integrals and their Implementation," BIT, v. 40, no. 4, 2004, pp. 755-772.

Johnson, W.P. (2002), " The Curious History of Faà di Bruno's Formula", Amer. Math. Monthly v. 109, 2002, pp.217-234.

Lagrange, G. L. (1768) Memoires de l'Acad.Roy. des Ssi. (Berlin) v. 24 (1768), p. 251

Landau, E. (1904) Berlin Sitzungberichte 1904 pp. 1118-33; Math. Zeitschrift v. 30 (1929) pp 616-17.

Lighthill, M. J. (1958) Introduction to Fourier Analysis and Generalized Functions, Cambridge Univ. Press, New York, 1958; 1960. MR 19, 1066; MR 22 \# 5888.

Lyness, J. N. (1971) "Adjusted forms of the Fourier coefficient asymptotic expansion and application in numerical quadrature," Math. of Comp., v. 25, no. 113, 1971, pp. 87-104.

Lyness, J. N. (2008) "Numerical Evaluation of a Fixed-Amplitude Variable -Phase Integral," Numer. Algor., v. 49, 2008, pp. 235-249.

Olver, F.W.J. (1974) Asymptotics and Special Functions, Academic Press, New York.

Whittaker, E.T. and G.N Watson (1953) A Course in Modern Analysis, Cambridge University Press. 
The following government license should be removed before publication.

The submitted manuscript has been created by UChicago Argonne, LLC, Operator of Argonne National Laboratory ("Argonne"). Argonne, a U.S. Department of Energy Office of Science laboratory, is operated under Contract No. DE-AC02-06CH11357. The U.S. Government retains for itself, and others acting on its behalf, a paid-up nonexclusive, irrevocable worldwide license in said article to reproduce, prepare derivative works, distribute copies to the public, and perform publicly and display publicly, by or on behalf of the Government. 